\title{
Analyse d'indicateurs de mesure pour les éclosions de maladies entériques, Centre de contrôle des maladies de la Colombie-Britannique, 2005-2014
}

\author{
D Fong ${ }^{1,2^{\star}}$, M Otterstatter ${ }^{1,3}$, M Taylor $^{1}$, E Galanis $^{1,3}$
}

\section{Résumé}

Contexte : Lors d'éclosions de maladies entériques, l'efficacité du contrôle dépend de la rapidité de l'intervention. La collecte systématique de données mesurables sur l'identification des éclosions, les enquêtes à leur sujet et leur contrôle peuvent aider à évaluer et à améliorer les interventions et éclairer ultérieurement les analyses et la modélisation de l'efficacité des interventions.

Objectif : Analyser les données sur les éclosions de maladies entériques en Colombie-Britannique, générer des indicateurs de mesure pour ces éclosions et évaluer leur utilisation pour la détermination de l'impact des interventions menées en réponse aux éclosions.

Méthodologie : Dans le cadre de cette étude descriptive, nous avons analysé les données de 57 enquêtes sur des éclosions provinciales et nationales de maladies entériques ayant entraîné la participation du Centre de contrôle des maladies de la Colombie-Britannique de 2005 à 2014. Les données ont été extraites de dossiers internes et de la base de données du Réseau canadien de renseignements sur la santé publique. Les indicateurs qui ont été analysés étaient les suivants : nombre de jours avant le lancement de l'enquête, nombre de jours avant l'intervention, nombre et types d'interventions, durée de l'enquête, durée de l'éclosion et nombre total de cas.

Résultats : Le délai médian avant le lancement d'une enquête sur une éclosion était de 36 jours et la durée médiane des enquêtes était de 39 jours. La durée médiane des éclosions était de 40 jours et le délai médian avant l'intervention était de 10 jours. La détermination de la source était associée à la réalisation d'une ou de plusieurs interventions $(P<0,0001)$. La durée des éclosions était corrélée avec le nombre de jours avant le lancement de l'enquête $\left(r_{s}=0,72\right.$, $P<0,0001)$ et avec le nombre de jours avant l'intervention $\left(r_{s}=0,51, P=0,025\right)$.

Conclusion : L'identification et l'analyse des indicateurs de mesure des éclosions permettent d'établir des niveaux de référence pouvant être comparés à ceux d'autres juridictions. Cela peut favoriser l'amélioration continue de la qualité et accroître les connaissances sur les effets des activités de santé publique. II est essentiel de recueillir des renseignements sur les dates de prise des mesures de santé publique afin de pouvoir évaluer la rapidité et l'efficacité des interventions en réponse aux éclosions.

\section{Affiliations}

${ }^{1}$ Centre de contrôle des maladies de la Colombie-Britannique, Vancouver (Colombie-Britannique)

${ }^{2}$ Centre de collaboration nationale en santé environnementale, Vancouver (Colombie-Britannique)

${ }^{3}$ École de santé publique et de santé des populations, Université de la Colombie-Britannique, Vancouver (Colombie-Britannique)

*Correspondance : daniel.fong@ bccdc.ca
Citation proposée : Fong D, Otterstatter M, Taylor M, Galanis E. Analyse d'indicateurs de mesure pour les éclosions de maladies entériques, Centre de contrôle des maladies de la Colombie-Britannique, 2005-2014. Relevé des maladies transmissibles au Canada. 2017;43(1):1-6. https://doi.org/10.14745/ccdr.v43i01a01f

\section{Introduction}

L'objectif d'une enquête sur une éclosion est d'en déterminer la source et de mener rapidement des interventions appropriées pour la contrôler (1). La rapidité des mesures de contrôle dépend souvent de la vitesse à laquelle une éclosion est décelée et expliquée. Si la mise en œuvre des mesures de contrôle est retardée, ces mesures sont susceptibles d'avoir peu de répercussions. Par conséquent, il est utile de faire le suivi d'indicateurs de mesure (qui peuvent être comparés au fil du temps et avec les données d'autres juridictions) liés à la rapidité des activités d'identification, d'enquête et de contrôle concernant les éclosions. Il est possible d'utiliser les données historiques sur les éclosions pour établir ces indicateurs de mesure. 
La collecte et l'analyse systématiques de données sur les indicateurs de mesure des éclosions peuvent éclairer les activités d'amélioration de la qualité. Aux États-Unis, le programme des Foodborne Diseases Centers for Outbreak Response Enhancement (FoodCORE) recueille des données de mesure normalisées sur les éclosions de maladies d'origine alimentaire afin d'améliorer les interventions liées aux éclosions (2). Des données sur les éclosions ont également été utilisées pour évaluer les effets des interventions. Seto et al. (2007) ont utilisé les données sur une éclosion d'Escherichia coli O157:H7 survenue dans plusieurs États aux États-Unis pour modéliser des stratégies de contrôle et ils ont constaté qu'une réduction de la transmission secondaire de 1 à $25 \%$ pouvait prévenir de 2 à $3 \%$ des cas secondaires et de 5 à $11 \%$ des sujets infectés et symptomatiques (3). Chen et al. (2014) ont utilisé les données sur une éclosion de shigellose d'origine hydrique dans une école chinoise pour examiner l'effet et la combinaison optimale de cing interventions sur le taux d'attaque et la durée de l'éclosion (4). Malgré l'utilité des indicateurs de mesure des éclosions, ces indicateurs sont rarement évalués ou signalés (5).

Le Centre de contrôle des maladies de la Colombie-Britannique (BCCDC) est responsable de la coordination des enquêtes sur les éclosions de maladies entériques qui touchent plus d'une région en Colombie-Britannique. II collabore également aux enquêtes sur les éclosions qui touchent une seule région de la Colombie-Britannique ou qui touchent cette province et plusieurs autres provinces canadiennes. Cela comprend la détermination des éclosions, l'élaboration de définitions de cas et de questionnaires sur les éclosions, l'analyse des données épidémiologiques recueillies, la mise en œuvre d'études épidémiologiques, la formulation de recommandations sur les mesures de contrôle et les communications avec le public (6). Les renseignements découlant de ces activités peuvent être utilisés pour améliorer les interventions de santé publique et leurs répercussions. II faudrait s'efforcer de concevoir et de mesurer des indicateurs qui seraient utiles pour les partenaires de la santé publique et pour le grand public.

Dans le présent article, nous rendons compte de la première phase d'une étude visant à évaluer l'effet des interventions sur la durée et l'ampleur des éclosions de maladies entériques. L'objectif de cette étude était d'analyser les données sur les éclosions de maladies entériques en Colombie-Britannique, de générer des indicateurs de mesure pour les éclosions et d'évaluer l'utilisation de ces indicateurs pour déterminer l'impact des interventions menées en réponse aux éclosions.

\section{Méthodologie}

Les données provenant d'enquêtes sur les éclosions de maladies entériques à l'échelle provinciale et nationale auxquelles le BCCDC avait participé ont été incluses dans l'analyse. Des données pour la période 2008-2014 ont été extraites de la base de données du Réseau canadien de renseignements sur la santé publique (RCRSP) le 24 javier 2015. Des données sur les éclosions entre 2005 et 2008 à l'égard desquelles le $B C C D C$ avait joué un rôle ont été extraites des dossiers internes du Centre, notamment des sommaires d'éclosions et des procès-verbaux de réunions liées aux enquêtes.

\section{Critères d'inclusion des éclosions}

Nous avons défini la participation du BCCDC selon deux critères : au moins un cas de maladie entérique était survenu en Colombie-Britannique et le BCCDC avait participé à l'enquête sur l'éclosion dans le cadre de réunions, en offrant un soutien épidémiologique et/ou en coordonnant l'enquête sur l'éclosion. Les éclosions de maladies entériques ont été incluses si elles correspondaient à la définition d'une éclosion au sein de la collectivité ( $\geq 2$ cas non liés d'une maladie similaire liés épidémiologiquement entre eux) ou d'une éclosion au sein d'un établissement ( $\geq 3$ cas d'une maladie similaire liés épidémiologiquement entre eux), ou si elles constituaient un cas unique de botulisme (d'après le manuel de l'utilisateur sur Sommaires d'éclosions, version 2, de l'Agence de la santé publique du Canada).

\section{Indicateurs de mesure des éclosions}

Dans les cas qui s'y prêtaient, les indicateurs de mesure des éclosions ont été définis selon les Guidelines for Foodborne Disease Outbreak Response établies par le Council to Improve Foodborne Outbreak Response (1). Dans les autres cas, les définitions correspondent à celles du dictionnaire de données du RCRSP (Gouvernement du Canada, dictionnaire de données pour les sommaires d'éclosions, module des maladies entériques et d'origine hydrique et alimentaire, Réseau canadien de renseignements sur la santé publique, s.d.).

Dans la mesure du possible, nous avons extrait ou calculé les données sur les indicateurs de mesure définis au tableau 1.

Tableau 1 : Définitions des principaux indicateurs de mesure des éclosions de maladies entériques

\begin{tabular}{|l|l|}
\hline \multicolumn{1}{|c|}{ Indicateur } & \multicolumn{1}{c|}{ Définition } \\
\hline $\begin{array}{l}\text { Jours avant le } \\
\text { lancement de } \\
\text { l'enquête }\end{array}$ & $\begin{array}{l}\text { Intervalle entre la date d'apparition des premiers } \\
\text { symptômes connus et la date de début de } \\
\text { l'enquête sur l'éclosion }\end{array}$ \\
\hline $\begin{array}{l}\text { Jours avant } \\
\text { l'intervention }\end{array}$ & $\begin{array}{l}\text { Intervalle entre la date de début de l'enquête sur } \\
\text { l'éclosion et la date de la première intervention, } \\
\text { le cas échéant }\end{array}$ \\
\hline $\begin{array}{l}\text { Durée de } \\
\text { l'enquête }\end{array}$ & $\begin{array}{l}\text { Intervalle, en jours, entre la date de début et la } \\
\text { date de fin de l'enquête sur l'éclosion }\end{array}$ \\
\hline $\begin{array}{l}\text { Durée de } \\
\text { l'éclosion }\end{array}$ & $\begin{array}{l}\text { Intervalle, en jours, entre la date d'apparition } \\
\text { des premiers symptômes connus et la date } \\
\text { d'apparition des derniers symptômes connus }\end{array}$ \\
\hline $\begin{array}{l}\text { Nombre total de } \\
\text { cas }\end{array}$ & $\begin{array}{l}\text { Nombre total de cas cliniques déclarés et de } \\
\text { cas confirmés en laboratoire dans le cadre de } \\
\text { l'éclosion }\end{array}$ \\
\hline
\end{tabular}

Les indicateurs temporels sont compatibles avec les événements d'une éclosion typique de maladie entérique (figure 1). D'autres variables telles que le nombre et les types d'interventions menées, l'agent étiologique, le mode de transmission, les renseignements sur la source, l'emplacement des cas et l'organisme déclarant ont servi à mettre les résultats en contexte. 
Figure 1 : Progression d'une éclosion de maladie entérique et indicateurs de mesure temporels

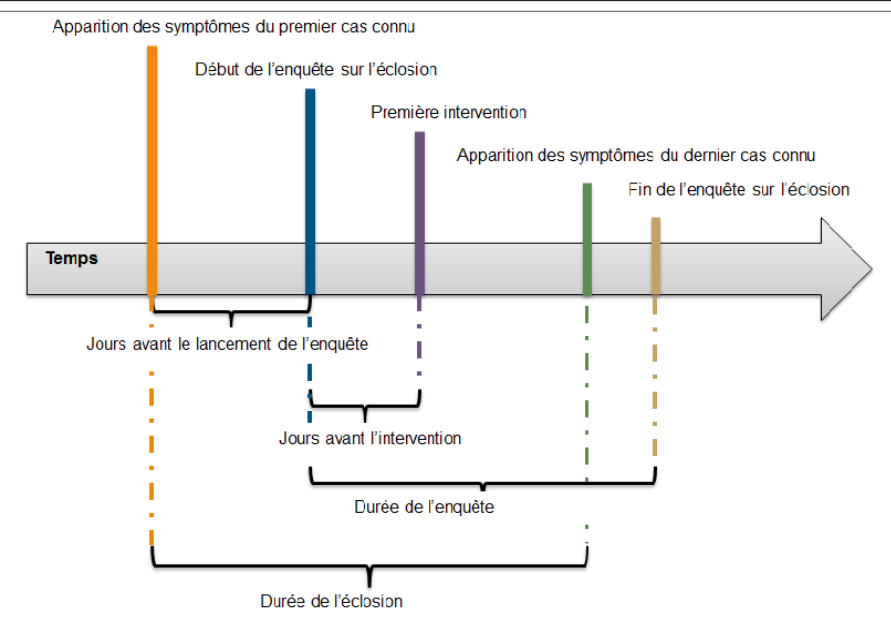

Une intervention a été définie comme étant une mesure de santé publique qui a pour but d'éliminer ou de réduire l'exposition à la source de l'éclosion ou de réduire la vulnérabilité d'une personne à l'infection. Les types d'intervention comprenaient des mesures applicables dans des établissements (fermeture, exclusion de membres du personnel, désinfection), ainsi que des mesures de sensibilisation, d'immunisation, de modification des politiques, de diffusion de communiqués de presse et de rappel de produits.

Les éclosions ont été décrites au moyen du nombre de cas et de jours, ainsi que des médianes et des plages de valeurs. Des analyses inférentielles ont été effectuées pour dégager des relations préliminaires entre les activités d'enquête sur l'éclosion, le moment de ces activités et/ou le nombre de cas; ces analyses avaient pour objet d'éclairer les approches d'évaluation de l'efficacité des interventions dans le cadre d'études futures. La méthode exacte de Fisher a été utilisée pour vérifier la signification statistique de la relation entre la connaissance de la source de l'éclosion et le recours aux interventions. La corrélation de rang de Spearman a été utilisée pour vérifier la signification statistique des relations entre les indicateurs temporels (durée) et le nombre de cas. Les analyses ont été effectuées à l'aide de la version 3.2.2 du progiciel R de calcul statistique et de Microsoft Excel 2010.

\section{Résultats}

\section{Indicateurs de mesure des éclosions}

Les caractéristiques des éclosions sont résumées au tableau 2. Parmi les 57 éclosions de maladies entériques ayant entraîné la participation du BCCDC entre 2005 et 2014, la grande majorité ( $88 \%, n=50$ ) présentaient des cas situés sur le territoire de plus d'une régie régionale de la santé et la plupart (79\%, n = 45) étaient d'origine alimentaire. Le nombre médian de cas d'éclosion de Salmonella, de cas d'infection à E. coli O157:H7 et de cas totaux était de 22,5 (intervalle : de 3 à 1 029), 16 (intervalle : de 3 à 85) et 18 (intervalle : de 1 à 1 029), respectivement. Salmonella et $\mathrm{E}$. coli $\mathrm{O} 157: \mathrm{H} 7$ ont été mises en cause dans $63 \%(n=36)$ des éclosions et ont contribué à $76 \%$
Tableau 2 : Caractéristiques des éclosions de maladies entériques ayant entraîné la participation du Centre de contrôle des maladies de la Colombie-Britannique, selon l'étiologie, 2005-2014

\begin{tabular}{|c|c|c|c|c|c|}
\hline \multirow{2}{*}{\multicolumn{2}{|c|}{$\begin{array}{c}\text { Indicateur } \\
\mathrm{N}(\%)\end{array}$}} & Salmonella & E. coli 0157:H7 & Autre $^{1}$ & \multirow{2}{*}{ Total } \\
\hline & & $\mathbf{N}(\%)$ & N (\%) & $\mathbf{N}(\%)$ & \\
\hline \multicolumn{2}{|l|}{ Éclosions } & $24(42)$ & $12(21)$ & $21(37)$ & $\begin{array}{r}57 \\
(100)\end{array}$ \\
\hline \multicolumn{2}{|l|}{ Cas } & $2025(67)$ & $266(9)$ & $\begin{array}{l}742 \\
(25)\end{array}$ & $\begin{array}{r}3033 \\
(100)\end{array}$ \\
\hline \multirow{4}{*}{ Lieu } & International & $7(12)$ & $3(5)$ & $1(2)$ & 11 (19) \\
\hline & $\begin{array}{l}>1 \text { province ou } \\
\text { territoire }\end{array}$ & $9(16)$ & $8(14)$ & $8(14)$ & $25(44)$ \\
\hline & $>1$ USS/RRS & $8(14)$ & $1(2)$ & $5(9)$ & $14(25)$ \\
\hline & 1 USS/RRS & 0 & 0 & $7(12)$ & $7(12)$ \\
\hline \multirow{3}{*}{$\begin{array}{l}\text { Mode de } \\
\text { transmission }\end{array}$} & $\begin{array}{l}\text { D'origine } \\
\text { alimentaire }\end{array}$ & $18(32)$ & $10(18)$ & $17(30)$ & 45 (79) \\
\hline & Autres $^{2}$ & $3(5)$ & 0 & $1(2)$ & $4(7)$ \\
\hline & Inconnu & $3(5)$ & $2(4)$ & $3(5)$ & $8(14)$ \\
\hline \multirow{2}{*}{ Source } & Connu $^{3}$ & $12(21)$ & $6(11)$ & $8(14)$ & $26(46)$ \\
\hline & Inconnu & $12(21)$ & $6(11)$ & $13(23)$ & $31(54)$ \\
\hline \multirow[b]{2}{*}{ Interventions } & $>1$ intervention & $14(25)$ & $6(11)$ & $7(12)$ & $27(47)$ \\
\hline & Total & $31(54)$ & $12(21)$ & $14(25)$ & $\begin{array}{r}57 \\
(100)\end{array}$ \\
\hline
\end{tabular}

Abréviations : N, nombre; USS/RRS, unité de services de santé/régie régionale de la santé; \%, pourcentage

Cyclospora $(n=5)$, Clostridium botulinum $(n=4)$, Campylobacter $(n=3)$, virus de l'hépatite A $(n=3)$, intoxication par phycotoxine (diarrhéique/paralysante, $n=2)$, Shigella $(n=2)$, Listeria $(n=1)$ et norovirus $(n=1)$

${ }^{2} \mathrm{D}$ 'animal à personne, de personne à personne ou par des friandises pour animaux de compagnie Les sources identifiées étaient principalement des aliments $(42,1 \%, n=24)$, notamment la viande, les légumes/fruits, les fruits de mer, les œufs, les condiments, les graines/noix légumineuses et les produits laitiers. Les friandises pour animaux de compagnie ont été mises en cause dans deux éclosions $(3,5 \%)$

( $n=2291)$ des cas liés aux éclosions. Au cours de la période à l'étude, il y a eu un nombre médian de six éclosions par année donnant lieu à une enquête (intervalle : 3 à 8 ) et près de la moitié des éclosions ( $47 \%, \mathrm{n}=27$ ) sont survenues au cours de l'été (juin à août). La source a été identifiée pour $46 \%(n=26)$ des éclosions.

Les indicateurs temporels de mesure des éclosions sont résumés au tableau 3 . Des données sur les éclosions étaient généralement disponibles pour le calcul des jours avant le lancement de l'enquête $(93 \%, \mathrm{n}=53)$, pour la durée de l'enquête $(77 \%, \mathrm{n}=44)$ et pour la durée de l'éclosion (95\%, $\mathrm{n}=54)$. Le délai médian avant le début d'une enquête sur une éclosion était de 36 jours et la durée médiane des enquêtes était de 39 jours. Les éclosions présentant un bref délai avant le début de l'enquête avaient une étendue géographique moindre. Par exemple, $85 \%(n=11 / 13)$ des enquêtes entreprises dans un délai de 18 jours ( $1^{\text {er }}$ quartile) concernaient une seule province, tandis que $92 \%(n=11 / 12)$ des enquêtes amorcées après 55 jours ( $3^{e}$ quartile) concernaient des éclosions comptant des cas dans plusieurs provinces/territoires. En outre, les éclosions à bref délai avant l'enquête étaient celles dont les symptômes ou agents étiologiques étaient distincts et avaient de courtes périodes d'incubation, ou celles qui ne nécessitaient pas de sous-typage moléculaire pour l'établissement d'un lien (p. ex. intoxication à la phycotoxine paralysante, Clostridium botulinum et norovirus). À l'opposé, les agents étiologiques à longue période d'incubation comme le virus de l'hépatite A et Listeria étaient associés à des éclosions où l'enquête avait été entreprise dans un délai plus long que la médiane de 36 jours. 
Tableau 3 : Indicateurs temporels des éclosions de maladies entériques ayant entraîné la participation du Centre de contrôle des maladies de la ColombieBritannique, 2005-2014

\begin{tabular}{|c|c|c|c|c|c|}
\hline Indicateur & Médiane & $1^{\text {er }}$ quartile & $3^{e}$ quartile & Intervalle & $\mathbf{N}$ \\
\hline $\begin{array}{l}\text { Jours avant le } \\
\text { lancement de } \\
\text { l'enquête }\end{array}$ & 36 & 18 & 55 & $0-620$ & 53 \\
\hline $\begin{array}{l}\text { Durée de } \\
\text { l'enquête (jours) }\end{array}$ & 39 & 20 & 78 & $0-1651$ & 44 \\
\hline $\begin{array}{l}\text { Durée de } \\
\text { l'éclosion (jours) }\end{array}$ & 40 & 16 & 84 & $0-1689$ & 54 \\
\hline $\begin{array}{l}\text { Jours avant } \\
\text { l'intervention - } \\
\text { première } \\
\text { intervention }\end{array}$ & 10 & 4 & 25 & $1-106$ & 20 \\
\hline $\begin{array}{l}\text { Jours avant le } \\
\text { communiqué de } \\
\text { presse }\end{array}$ & 6 & 4 & 33 & $2-140$ & 14 \\
\hline $\begin{array}{l}\text { Jours avant le } \\
\text { rappel de produits }\end{array}$ & 9 & 4 & 19 & $2-106$ & 16 \\
\hline $\begin{array}{l}\text { Jours avant } \\
\text { l'intervention - } \\
\text { autres } \\
\text { interventions }{ }^{1}\end{array}$ & 13 & 10 & 25 & $1-74$ & 6 \\
\hline
\end{tabular}

Abréviation : N, nombre

'Fermeture de l'installation, sensibilisation, exclusion de membres du personnel, immunisation des personnes vulnérables

\section{Indicateurs de mesure des interventions en réponse aux éclosions}

Près de la moitié (47 \%, $\mathrm{n}=27$ ) des éclosions avaient fait l'objet d'au moins une intervention déclarée; la plupart d'entre elles $(70 \%, n=19)$ n'avaient pas donné lieu à plus de deux interventions (tableau 2). Le nombre médian des interventions par éclosion pour Salmonella et E. coli O157:H7, et pour toutes les éclosions, était de 1 (intervalle : de 0 à 5), 0,5 (intervalle : de 0 à 3 ) et 0 (intervalle : de 0 à 5), respectivement. Sur un total de 57 interventions documentées, la plupart $(75 \%, n=43)$ étaient associées à Salmonella ou à E. coli O157:H7.

L'identification de la source était significativement associée à la mise en œuvre d'une ou de plusieurs interventions $(P<0,0001)$. Les rappels de produits, la fermeture de l'établissement, la désinfection de l'établissement et l'immunisation étaient des interventions mises en œuvre uniquement lorsque la source avait été identifiée, alors que les communiqués de presse, la sensibilisation, l'exclusion de membres du personnel et la modification des politiques étaient des interventions mises en œuvre peu importe que la source ait été identifiée ou non (figure 2). Outre les rappels de produits et les communiqués de presse, les autres types d'intervention étaient peu souvent signalés ou leur date de mise en œuvre n'était pas disponible; dans le cas de 21 (37\%) des 57 interventions, il n'y avait pas suffisamment de renseignements pour qu'il soit possible de calculer le délai avant l'intervention. Parmi les 27 éclosions ayant donné lieu à au moins une intervention, il y avait suffisamment de renseignements sur $74 \%$ des cas $(n=20)$ pour qu'il soit possible de calculer le délai avant l'intervention. Le délai médian avant l'intervention était de 10 jours; le délai médian des rappels de produits était de neuf jours après le début de l'enquête, et celui des communiqués de presse, de six jours après le début de l'enquête (tableau 3).
Figure 2 : Fréquence et types d'interventions menées, selon que la source de l'éclosion était connue ou non $(n=57)$

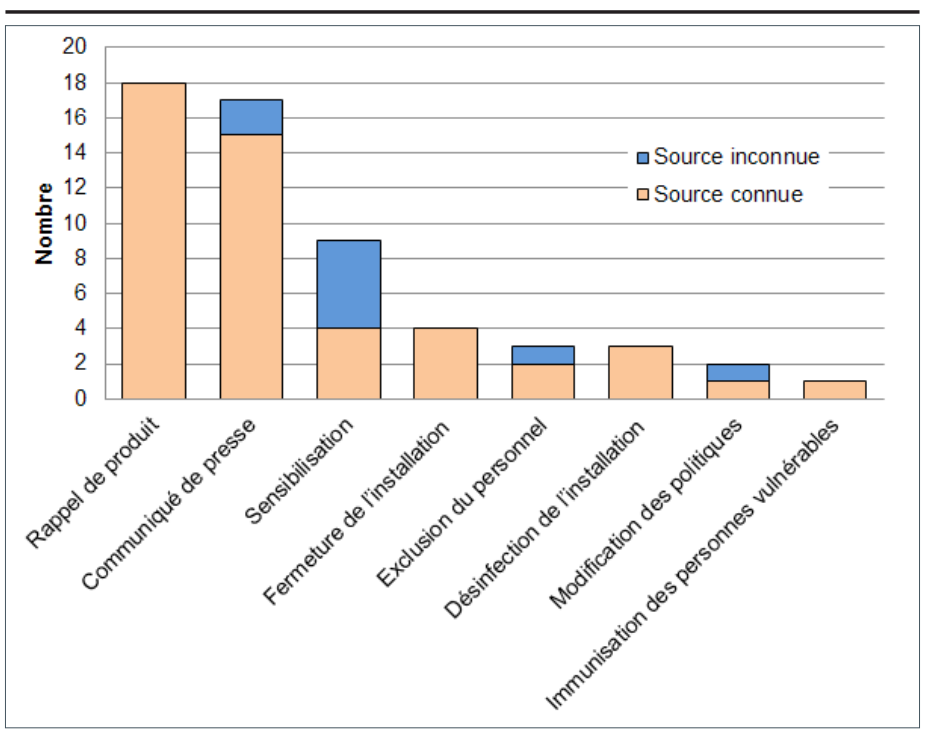

La durée des éclosions était positivement corrélée avec le nombre de jours avant le lancement de l'enquête $\left(r_{s}=0,72, P<0,0001\right)$ et avec le nombre de jours avant I'intervention $\left(r_{s}=0,51, P=0,025\right)$. Le nombre de jours avant I'intervention était aussi positivement corrélé avec le nombre de jours avant le lancement de l'enquête $\left(r_{s}=0,47, P=0,036\right)$. II n'y avait pas de corrélation significative entre le nombre total de cas et le nombre de jours avant le lancement de l'enquête ou de jours avant l'intervention $\left(r_{s}=0,16, P=0,27 ; r_{s}=-0,082\right.$, $P=0,73$, respectivement).

\section{Discussion}

À l'époque de la présente étude, nous n'avions connaissance d'aucune analyse déjà publiée concernant des indicateurs de mesure liés aux éclosions de maladies entériques et aux interventions connexes au Canada. Nous fournissons des renseignements sur des indicateurs de mesure pour les éclosions qui peuvent être utilisés afin d'évaluer la pertinence du moment des enquêtes et des interventions effectuées en cas d'éclosion de maladies entériques.

\section{Réalisation des interventions}

Nos résultats indiquent que l'identification de la source d'une éclosion était associée à la prise de mesures d'intervention. Pour certaines interventions (p. ex. rappel de produits), I'identification de la source est nécessaire, car ces interventions éliminent la source en cause. Les interventions non spécifiques (p. ex. sensibilisation) peuvent être effectuées sans que l'on ait de certitude absolue quant à la source. Nous avons constaté que les rappels de produits, les communiqués de presse et la sensibilisation étaient des interventions mises en œuvre plus fréquemment que les fermetures d'établissement ou l'exclusion des personnes malades, ce qui est compatible avec le fait que les éclosions étudiées tendaient à affecter des régions étendues au lieu d'être localisées ou de se limiter à un établissement. Le programme FoodCORE a rapporté qu'entre 2010 et 2012, la 
source avait été identifiée dans une moyenne de $30 \%$ des éclosions de Salmonella, d'E. coli O157:H7 et de Listeria (2). Dans le cadre de notre étude, près de la moitié des éclosions avaient une source connue (tableau 2). Cette différence est peut-être due aux critères plus rigoureux applicables à la tenue d'enquêtes sur les éclosions en Colombie-Britannique depuis 2011; ces critères ont été établis de telle sorte que l'accent soit mis sur les éclosions dont la source pourrait être plus facilement identifiée (7). L'identification de la source est un jalon d'une importance cruciale pour la prise de mesures de contrôle ciblées.

\section{Durée des éclosions et nombre total de cas}

Nos résultats indiquent que plus le nombre de jours avant le lancement de l'enquête augmente, plus l'éclosion est longue et plus le nombre de jours avant l'intervention est élevé. De même, plus le nombre de jours avant l'intervention augmente, plus la durée de l'éclosion s'accroît. Dans les cas où les interventions sont retardées, la transmission de la maladie se poursuit, ce qui entraîne de plus longues éclosions. Nos résultats préliminaires indiquent une corrélation entre la rapidité des activités en réponse à une éclosion et la durée de l'éclosion. Il faudra effectuer des recherches supplémentaires pour confirmer ces relations.

Le suivi régulier des indicateurs de mesure des éclosions permet de faire des comparaisons entre les niveaux de référence internes et externes au fil du temps, et de déceler des activités qui engendrent des améliorations. Le programme FoodCORE rapporte depuis 2011 des indicateurs annuels de mesure des éclosions visant à permettre l'évaluation des activités de détection, d'intervention et de contrôle liées aux éclosions (8-11). Il fait état d'une durée médiane de l'enquête (calculée de la date de notification de la grappe à la date de fin de l'enquête) de 26 à 35 jours pour Salmonella (12-14); d'après nos données, nous avons constaté que la durée médiane de l'enquête était de 49 jours. Cette différence peut être due au fait que notre ensemble de données comprenait plusieurs éclosions d'envergure (> 100 jours), dont une éclosion de $S$. Enteritidis qui a duré quatre ans. Les différences opérationnelles entre les activités d'intervention et de surveillance peuvent être une autre cause des durées différentes observées. De telles différences inhérentes dans les sources de données et dans les modalités de mise en œuvre peuvent limiter les comparaisons externes, mais les comparaisons internes au fil du temps sont d'une précieuse utilité pour le suivi des améliorations. La collecte de données sur les indicateurs de mesure liés aux éclosions et les rapports sur leurs modes d'utilisation comme indicateurs du rendement permettront une évaluation et une analyse plus riche des tendances, notamment pour ce qui est de la rapidité des interventions.

Bien que notre analyse n'ait pas permis de dégager une relation significative à cet égard, on peut s'attendre à ce que le nombre total de cas diminue si les enquêtes ou les interventions surviennent plus tôt. Un examen d'enquêtes sur des éclosions en Europe n'a produit aucune corrélation entre la rapidité de la réalisation d'une étude analytique de l'éclosion et le nombre total de cas (5). Malgré tout, les modèles indiquent que des retards dans la déclaration du cas de référence aux autorités de santé publique augmentent la proportion des infections prévues causées par le cas de référence et les cas secondaires (15). Le nombre de cas est probablement influencé par d'autres facteurs liés à la dynamique de la transmission.

\section{Limites des données et incidences pour les recherches futures}

L'absence de renseignements sur les dates d'un grand nombre d'interventions limite la capacité d'effectuer des analyses plus poussées. Les données regroupées sont faussées par la forte proportion des éclosions de Salmonella et d'E. coli O157:H7; des statistiques sommaires n'ont pas pu être calculées pour de nombreux autres agents étiologiques.

Les enquêtes sur les éclosions peuvent suivre un ordre d'activités logique, mais nos données étaient largement de nature transversale, ce qui a limité les inférences précises concernant les causes et les effets. Le nombre de cas et les indicateurs de mesure temporels variaient considérablement et l'efficacité statistique était limitée par la petite taille de l'échantillon. Les éclosions déclarées par les autorités sanitaires régionales à propos desquelles le BCCDC n'est pas intervenu ont été exclues parce que leur portée était opérationnellement distincte (p. ex. différents milieux, modes de gestion) et que le BCCDC n'avait pas accès aux données requises. Par conséquent, les résultats présentés peuvent s'appliquer uniquement aux situations d'éclosion à grande échelle (provinciale ou nationale).

Depuis 2011, le BCCDC a établi des critères qui prennent en compte un nombre minimal des cas et leur répartition géographique dans la décision de lancer une enquête sur une éclosion de maladies entériques (7). Dans nos résultats, nous observons que cela se reflète dans la forte proportion d'éclosions de Salmonella et d'E. coli O157:H7 d'origine alimentaire touchant de multiples provinces/territoires, ainsi que dans la faible proportion d'éclosions localisées attribuables à des agents étiologiques à brève période d'incubation, et principalement propagées de personne à personne (p. ex. norovirus). Par conséquent, tout indicateur temporel de mesure des éclosions et toute intervention connexe doivent être interprétés dans le contexte des types d'éclosion inclus dans l'analyse.

Une documentation exhaustive et uniforme sur les éclosions, y compris les listes détaillées complètes et les dates des jalons de chaque éclosion, serait nécessaire pour qu'il soit possible d'examiner de façon plus approfondie I'incidence des interventions. II serait utile de disposer de données additionnelles et de procéder à des modélisations mathématiques pour examiner les liens entre la rapidité des interventions, la durée des éclosions, le nombre de cas au fil du temps et le nombre escompté de cas évités. La modélisation pourrait aussi permettre d'évaluer des stratégies d'intervention combinées reflétant plus fidèlement les situations réelles. Se fondant sur des données relatives à des éclosions antérieures, des études ont eu recours à des modèles pour simuler la répartition temporelle prévue des cas (courbe épidémique) et quantifier l'effet des interventions $(3,4)$.

\section{Conclusion}

La présente étude décrit comment il est possible d'utiliser les données sur les éclosions pour mettre au point des indicateurs de mesure et les utiliser afin d'évaluer l'opportunité des 
6. Disponible à l'adresse : http://wwwnc.cdc.gov/eid/ article/13/6/06-1264_article.

4. Chen T, Leung RK, Zhou Z, Liu R, Zhang X, Zhang L. Investigation of key interventions for shigellosis outbreak control in China. PLoS ONE. 2014;9(4):e95006. Disponible à I'adresse : doi.org/10.1371\%2Fjournal.pone.0095006.

5. van de Venter E, Oliver I, Stuart JM. Timeliness of epidemiological outbreak investigations in peer-reviewed European publications, January 2003 to August 2013. Euro Surveill. 2015;20(6).

6. BC Centre for Disease Control. Communicable diseases prevention - Communicable Diseases Prevention and Control Services (CDPACS) is an integrated mix of programs [Internet]. Vancouver: BCCDC [consulté le 4 nov 2016]. Disponible à I'adresse : http://www.bccdc.ca/our-services/service-areas/ communicable-diseases-prevention.

7. Taylor M, Galanis E. Établissement de critères pour lances des enquêtes sur les éclosions de maladies entériques en Colombie Britannique. Relevé des maladies transmissibles au Canada [Internet]. 2014 [consulté le 4 nov 2016];40(Suppl 1):10-6. Disponible à l'adresse : http://www.phac-aspc.gc.ca/ publicat/ccdr-rmtc/14vol40/dr-rm40s-1/dr-rm40s-1-interv-fra. php.

8. Centers for Disease Control and Prevention. FoodCORE Salmonella, Shiga toxin-producing E. coli, and Listeria metrics rationale and intent [Internet]. Atlanta (GA): CDC [mis à jour le 17 fév 2016; consulté le 4 nov 2016]. Disponible à l'adresse : http://www.cdc.gov/foodcore/pdfs/ssl-rationale-intent.pdf.

9. Centers for Disease Control and Prevention. FoodCORE model practice: initial case-patient interviewing [Internet]. Atlanta (GA): CDC [mis à jour le 30 sept; consulté le 4 nov 2016]. Disponible à l'adresse : http://www.cdc.gov/foodcore/ pdfs/247981_foodcore-patient-interviewing_508.pdf.

10. Centers for Disease Control and Prevention. FoodCORE model practice: laboratory timeliness and completeness [Internet]. Atlanta (GA): CDC; [mars 2014; consulté le 4 nov 2016]. Disponible à l'adresse : http://www.cdc.gov/foodcore/ pdfs/245823_foodcore-model-practice-factsheet_508.pdf.

11. Centers for Disease Control and Prevention. About FoodCORE [Internet]. Atlanta (GA): CDC; 2014 [mis à jour le 30 sept; consulté le 4 nov 2016]. Disponible à l'adresse : http://www.cdc.gov/foodcore/about.html.

12. Centers for Disease Control and Prevention. CDC FoodCORE year one cumulative metrics data - Salmonella, Shiga toxinproducing Escherichia coli, and Listeria (SSL). Report period: October 1, 2010 to September 30, 2011 [Internet]. Atlanta (GA): CDC; [mis à jour le 16 août 2013; consulté le 4 nov 2016]. Disponible à l'adresse : http://www.cdc.gov/foodcore/ metrics/metrics-table-year-1-ssl.html.

13. Centers for Disease Control and Prevention. CDC FoodCORE year three cumulative metrics - Salmonella, Shiga toxinproducing Escherichia coli, and Listeria (SSL). Data report period: January 1, 2013 to December 31, 2013 [Internet]. Atlanta (GA): CDC; [mis à jour le 17 fév 2016; consulté le 4 nov 2016]. Disponible à l'adresse : http://www.cdc.gov/ foodcore/metrics/metrics-table-year-3-ssl.html.

14. Centers for Disease Control and Prevention. CDC FoodCORE year two cumulative metrics - Salmonella, Shiga toxinproducing Escherichia coli, and Listeria (SSL). Data report period: October 1, 2011 to December 31, 2012 [Internet]. Atlanta (GA): CDC; [mis à jour le 17 fév 2016; consulté le 4 nov 2016]. Disponible à l'adresse : http://www.cdc.gov/ foodcore/metrics/metrics-table-year-2-ssl.html.

15. Bonacic Marinovic A, Swaan C, van Steenbergen J, Kretzschmar M. Quantifying reporting timeliness to improve outbreak control. Emerg Infect Dis. 2015;21(2):20916. Disponible à l'adresse : http://wwwnc.cdc.gov/eid/ article/21/2/13-0504_article. 